\section{Development of a handheld submersible chemiluminescent sensor: Quantification of superoxide at coral surfaces}

Kalina C. Grabb ${ }^{1,2}$, Jason Kapit ${ }^{3}$, Scott D. Wankel ${ }^{1}$, Kevin Manganini ${ }^{3}$, Amy Apprill ${ }^{1}$, Maickel Armenteros ${ }^{4}$, and Colleen M. Hansel ${ }^{1 *}$

${ }^{1}$ Woods Hole Oceanographic Institution, Marine Chemistry and Geochemistry, Woods Hole, MA, USA

${ }^{2}$ Department of Earth, Atmospheric and Planetary Sciences, Massachusetts Institute of Technology, 77 Massachusetts Avenue, Cambridge, MA USA

${ }^{3}$ Woods Hole Oceanographic Institution, Applied Ocean Physics and Engineering, Woods Hole, MA, USA

${ }^{4}$ Centro de Investigaciones Marinas, Universidad de La Habana. 16 \# 114, Playa, CP 11300 , Habana, Cuba

*Corresponding author: chansel@whoi.edu 

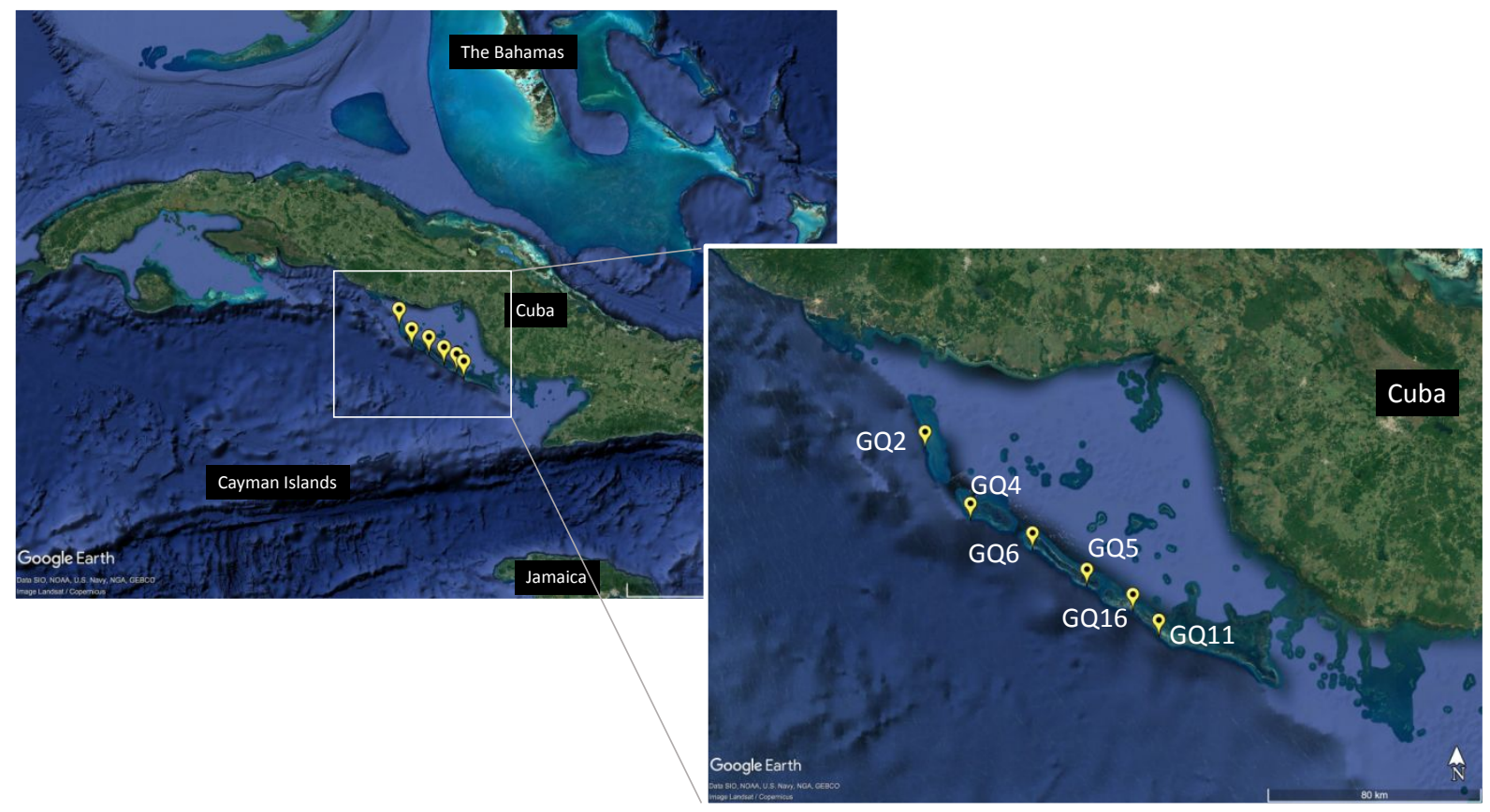

30 Figure S1. Location of sample sites in Jardines de la Reina, Cuba (yellow marker). Sites are

31 labeled and correspond to the sites where we conducted in situ extracellular superoxide

32 measurements.

33

34

35

36

37

38

39

40

41

42

43

44

45

46

47

48

49

50

51

52

53

54 


\begin{tabular}{|c|c|c|c|c|c|c|c|c|c|c|c|c|c|c|c|c|c|c|c|}
\hline $\begin{array}{c}\text { Sample } \\
\text { Date }\end{array}$ & Site & Latitude (N) & Longitude (W) & $\begin{array}{l}\overrightarrow{0} \\
\stackrel{\Xi}{\Xi} \\
\stackrel{0}{\circ}\end{array}$ & 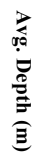 & 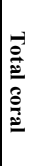 & $\begin{array}{c}\text { Species of } \\
\text { individuals } \\
\text { sampled }\end{array}$ & 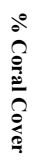 & 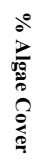 & 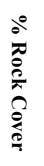 & 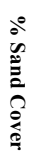 & 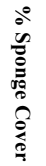 & $\begin{array}{l}\hat{0} \\
\hat{\varepsilon}\end{array}$ & $\stackrel{\text { Z }}{\widehat{E}}$ & $\begin{array}{c}\stackrel{\Xi}{0} \\
\stackrel{+}{3} \\
\stackrel{3}{3}\end{array}$ & 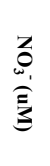 & $\begin{array}{l}\text { 孞 } \\
\text { 令 }\end{array}$ & $\begin{array}{l}\underline{Z} \\
\stackrel{\mathbf{E}}{\mathbf{E}} \\
\widehat{E}\end{array}$ & $\stackrel{\mathscr{E}}{\hat{\xi}}$ \\
\hline \multirow{2}{*}{20 -Nov-17 } & \multirow{2}{*}{ GQ2 } & \multirow{2}{*}{$21^{\circ} 18^{\prime} 12.0^{\prime \prime} \mathrm{N}$} & \multirow{2}{*}{$79^{\circ} 35^{\prime} 27.84^{\prime \prime} \mathrm{W}$} & \multirow[t]{2}{*}{-} & \multirow{2}{*}{9} & \multirow{2}{*}{4} & \multirow{2}{*}{$\begin{array}{l}\text { Dcyl (1), Mcav } \\
\text { (2), Ppor (1) }\end{array}$} & \multirow{2}{*}{11} & \multirow{2}{*}{41} & \multirow{2}{*}{44} & \multirow{2}{*}{0} & \multirow{2}{*}{3} & 71.5 & 5.5 & 0.16 & 0.01 & 0.05 & 0.27 & 1.3 \\
\hline & & & & & & & & & & & & & 69.6 & 4.6 & 0.20 & 0.04 & 0.06 & 0.16 & 1.3 \\
\hline \multirow{2}{*}{ 5-Nov-17 } & \multirow{2}{*}{ GQ4 } & \multirow{2}{*}{$21^{\circ} 03^{\prime} 49.5^{\prime \prime} \mathrm{N}$} & \multirow{2}{*}{$79^{\circ} 25^{\prime} 38.1 " \mathrm{~W}$} & \multirow{2}{*}{28} & \multirow{2}{*}{11} & \multirow{2}{*}{4} & \multirow{2}{*}{ Past (4) } & \multirow{2}{*}{28} & \multirow{2}{*}{35} & \multirow{2}{*}{11} & \multirow{2}{*}{22} & \multirow{2}{*}{4} & 72.2 & 3.1 & 0.17 & 0.14 & 0.03 & 0.47 & 1.9 \\
\hline & & & & & & & & & & & & & 73.7 & 4.6 & 0.18 & 0.11 & 0.02 & 0.12 & 1.7 \\
\hline \multirow{2}{*}{ 6-Nov-17 } & \multirow{2}{*}{ GQ5 } & \multirow{2}{*}{$20^{\circ} 57^{\prime} 55.44 " \mathrm{~N}$} & \multirow{2}{*}{$79^{\circ} 12^{\prime} 16.38^{\prime \prime} \mathrm{W}$} & \multirow[b]{2}{*}{30} & \multirow{2}{*}{12} & \multirow{2}{*}{3} & Dlab (1), Mcav & & & & & & 72.4 & 4.8 & 0.17 & 0.11 & 0.04 & 0.07 & 1.8 \\
\hline & & & & & & & (1), Past (1) & 30 & 24 & 28 & 8 & 10 & 66.1 & 3.2 & 0.17 & 0.27 & 0.04 & 0.06 & 1.8 \\
\hline 7 Nou 17 & GO6 & $20 \circ 50136770 \mathrm{~N}$ & $70^{\circ} 00^{\prime} 35$ 76"W & & & $=$ & Acer (1), Ofav & 37 & 18 & 33 & 8 & 4 & 72.6 & 5.5 & 0.17 & 0.06 & 0.04 & 0.13 & 1.6 \\
\hline 7-Nov-17 & GQ6 & $205036.72 \mathrm{~N}$ & $790035.76^{\circ} \mathrm{W}$ & 28 & 12 & 4 & $\begin{array}{l}\text { (1), Past (1), } \\
\text { Ppor (1) }\end{array}$ & 37 & 18 & 33 & 8 & 4 & 68.4 & 3.9 & 0.17 & 0.20 & 0.05 & 0.11 & 1.4 \\
\hline & & & & & & & Mcav (1), Ofav & & & & & & 65.9 & 6.4 & 0.16 & 0.08 & 0.04 & $\mathbf{0 . 0 7}$ & 1.3 \\
\hline 11-Nov-17 & GQ11 & $20^{\circ} 40^{2} 22.56^{\prime \prime} \mathrm{N}$ & $78^{\circ} 45^{\prime} 11.22^{\prime \prime} \mathrm{W}$ & 28 & 9 & 6 & $\begin{array}{l}\text { (2), Past (1), } \\
\text { Ppor (2) }\end{array}$ & - & - & - & - & - & 65.3 & 1.3 & 0.17 & 0.22 & 0.05 & 0.07 & 1.4 \\
\hline & & 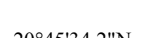 & $70050441 " 1 \mathrm{~T}$ & & & & Mcav (3), & & & P & & 5 & 67.3 & 1.1 & 0.16 & 0.10 & 0.03 & 0.06 & 1.3 \\
\hline Nov-17 & GQ16 & $20^{\circ} 45^{\prime} 34.2^{2} \mathrm{~N}$ & $785044 .{ }^{2} \mathrm{~W}$ & - & 14 & 6 & Mcav (3) & 23 & 28 & 42 & 2 & 5 & 67.3 & 6.6 & 0.16 & 0.20 & 0.05 & 0.42 & 1.5 \\
\hline
\end{tabular}

Table S1: Geographic, coral, and environmental data about the sample sites analyzed for in situ adult coral measurements in Jardines de la Reina, Cuba collected aboard the MV Alucia. All in situ data was collected on SCUBA. The coral species correspond with: Acropora cervicornis (Acer), Dendrogyra cylindricus (Dcyl), Diploria labyrinthiformis (Dlab), Montastraea cavernosa (Mcav), Orbicella faveolata (Ofav), Porites astreoides (Past), and Porites porites (Ppor). The number of samples is indicated within the parenthesis. Environmental data includes: Total Organic Carbon (TOC), Total Nitrogen (TN), phosphate $\left(\mathrm{PO}_{4}^{3-}\right)$, nitrate $\left(\mathrm{NO}_{3}{ }^{-}\right)$, nitrite $\left(\mathrm{NO}_{2}{ }^{-}\right)$, ammonium $\left(\mathrm{NH}_{4}{ }^{+}\right)$, and silicate (Si). Environmental data is shown for the surface depth (no highlight) and reef depth (highlight grey). 
89

90

91

92 